\title{
Fascinating friction
}

d $\mathrm{f}$ surfaces are slippery, it is usually because they are wet. This is the case even for ice: our skates glide smoothly across the ice because they glide across a thin layer of water. And so does a hockey puck. Water makes slippery, this much we know. So it should not come as a surprise that oil and grease also reduce friction. After all, they form a thin layer of fluid between two solid surfaces and thus provide lubrication.

Lubrication between dry surfaces can also arise if both surfaces are covered with patches of a well-ordered smooth layer of, e.g., graphene, but with the patches randomly oriented. Think of two washboards, which glide smoothly across one another at random orientations except if the ridges happen to be aligned.

But what about the friction between two ordinary dry surfaces? Let us first remember that the friction coefficient - the ratio between the friction force and the normal force - is smaller when the surfaces are moving relative to one another than when they

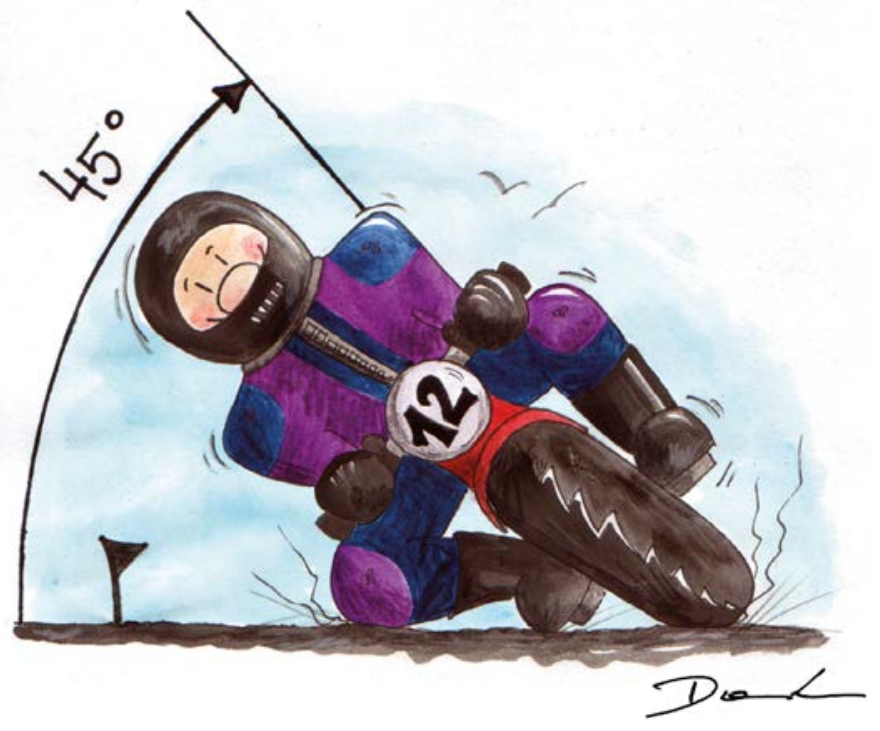

Can the friction coefficient reach values larger than 1 ? Why not? In fact, in some cases it does. And you know it does. Everybody who has seen motorcycle racing on TV has observed that, in curves, the bike can lean over by an angle larger than $45^{\circ}$ with respect to the surface normal. A simple force diagram shows us that, indeed, the static friction coefficient exceeds unity in this case, thanks to the silicone rubber or acrylic-rubber-coated tire surface.

Another striking property of friction, which most of us take for granted since our high-school physics, is the fact that the friction only depends on the normal force and the type of surfaces, but is independent of the surface area. If considered from a microscopic point of view, this flies in the face of common sense. The solution to this problem is that this 'area' is the apparent surface area. True atomic contact is established only in a small fraction of this area, even for polished surfaces. Elasticity of the contacting surfaces and/or their plastic response makes the true contact area approximately linear in the normal force. Problem solved.

It may be clear that much about friction is as yet poorly understood. Our present understanding is still at a semi-phenomenological level. Nobody has been able to predict the friction coefficient for a given system. Fortunately, the young and rapidly developing field of nanotribology is on course to clear up much of the friction mysteries, as is done for example by Joost Frenken and his group ${ }^{1}$ in Leiden/Amsterdam. And since friction is responsible for a substantial fraction of the cost of mechanical equipment in wear and energy use, this work may soon save some money in our daily life...

${ }^{1}$ The physics of atomic-scale friction: basic considerations and open questions; S.Yu. Krylov and J.W.M. Frenken, Review paper (invited), Phys. Status Solidi B 251, 711 (2014) 